\title{
3D object location and inversion of the magnetic polarisability tensor at a single frequency using a walk-through metal detector
}

\author{
Liam A Marsh ${ }^{1,3}$, Christos Ktistis ${ }^{1}$, Ari Järvi ${ }^{2}$, David W Armitage ${ }^{1}$ and \\ Anthony $\mathbf{J}$ Peyton ${ }^{1}$ \\ ${ }^{1}$ School of Electrical and Electronic Engineering, The University of Manchester, \\ Manchester, UK, M13 9PL \\ ${ }^{2}$ Rapiscan Systems Oy, Klovinpellontie 3, Torni 2, FI-02180 Espoo, Finland \\ E-mail: liam.marsh@manchester.ac.uk
}

\begin{abstract}
A tomographic metal detection and characterisation system has been designed and built for recovering information about magnetic and/or conductive objects within the detector space. This information is gathered as a result of a "walk-through" scan of a candidate in the same manner as for a typical security metal detector archway. Following the passage of the candidate, the system uses measurements from an array of coils to calculate the polarisability tensor, which describes the low frequency electromagnetic characteristic of a small metallic object when it interacts with an AC magnetic field. In addition to the magnetic polarisability dyadic tensor the position of the perturbation is also determined as a product of the inversion algorithm. The system has been tested and is capable of inverting object tensors with $<20 \%$ typical parameter variation, and determines three-dimensional object location with a typical error of less than $\pm 3 \mathrm{~cm}$. In this paper results are shown from a set of four different test object examples, each with a different magnetic polarisability tensor. This object set consists of a ferrite sphere, a ferrite rod, and phantom aluminium and steel handgun shapes.
\end{abstract}

\section{Introduction}

Walk-through metal detection (WTMD) has proven to be a fundamental part of the screening of large numbers of personnel for potential threat objects and WTMD use is widespread in locations such as airports, prisons, embassies, ports and government buildings. In these scenarios their primary purpose is to keep prohibited metallic items such as knives, guns and components of explosive devices out of a secured area.

Due to the importance of maintaining a high level of security, it is a natural assumption that walkthrough metal detectors should be as sensitive as possible. This is not the case for all scenarios, because there are a variety of operating requirements which depend upon the location in which the detector is being used. For example, at an airport passengers may be in possession of a variety of legitimate metallic items such as keys, mobile telephones, wristwatches and glasses, whereas in a prison it is possible to control the environment more strictly because inmates should not be in possession of any metallic items. Although the detector should be as sensitive as practically possible,

\footnotetext{
${ }^{3}$ To whom any correspondence should be addressed.
} 
there will usually be a large number of alarms triggered by the presence of innocuous items, where these are allowed.

The current technology of walkthrough metal detectors provides some basic location estimations for detected objects, which typically refer to a horizontal band within the detector. This basic localisation typically restricts the object to be within a particular 'horizontal band' of the detector space (typically $1 / 5$ to $1 / 10$ of the overall detector volume), as shown in figure 1 . As this is a relatively large area it can take a significant amount of time for an operator to search manually, and when this effect is multiplied across hundreds of candidates it can lead to significant delays. Methods such as this typically rely upon the heuristic analysis of peak signal responses from an array of coils, and relating these to their approximate locations. This is what gives rise to the zonal 'bands' that are typically used, i.e. the detector has a peak response at the same height as specified coils, therefore the object is assumed to be closest to them. Such systems are limited to a spatial resolution which is proportional to the number of coils employed within the system. The system presented in this paper both decreases the uncertainty of object positioning to the order of $<8 \mathrm{~cm}$, and also determines each position as a function of time, therefore allowing the possibility of tracking an object as it passes through the detector.

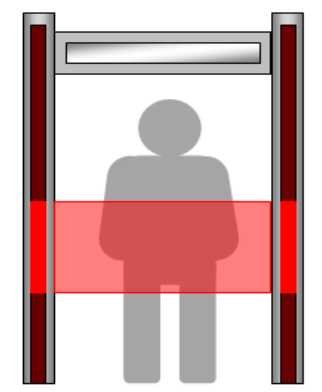

Figure 1. Typical degree of accuracy for a commercial WTMD.

Screening for contraband using WTMDs would be much more efficient in terms of the amount of time taken to scan a candidate, and also the rate of false positive alarms if greater information regarding the target object was made available. Such information may include the specific location, approximate dimensions and orientation of the threat object as well as additional material properties. Much of the object-specific information is contained in a quantity known as the "magnetic polarisability dyadic tensor', referred to as $\overleftrightarrow{\mathbf{M}}$. The double-arrowed accent is used to denote a dyadic quantity rather than a matrix or vector quantity. With these considerations in mind a prototype system has been constructed which allows for the inversion of the magnetic polarisability dyadic tensor, in addition to the 3D localisation of a threat object. The system can also operate with multiple metallic objects, however only the results for single objects will be reported in this paper.

The magnetic polarisability dyadic is a complex $3 \times 3$ matrix which defines the contribution of individual field components to the induced voltage at the receive coil. The theory introduced in section 2 shows that it is the only part of the theoretical description of the system which varies according to the object interacting with the field, and hence can be treated as a solo entity which can define the characteristics of the object in question [1]. A magnetic polarisability tensor can be used to characterise objects that alter the magnetic properties of the system e.g. a conductive or magnetic material. It is dependent upon excitation frequency, object conductivity and permeability [2]. It should also be noted that although the tensor contains 18 values (9 real and 9 imaginary), due to electromagnetic reciprocity between transmitted and received signals [3], the tensor is symmetric, and consequently only contains 12 independent values ( 6 real and 6 imaginary). The structure of the tensor is shown overleaf. 


$$
\overrightarrow{\mathbf{M}}=\left[\begin{array}{lll}
M_{11}+j N_{11} & M_{12}+j N_{12} & M_{13}+j N_{13} \\
M_{12}+j N_{12} & M_{22}+j N_{22} & M_{23}+j N_{23} \\
M_{13}+j N_{13} & M_{23}+j N_{23} & M_{33}+j N_{33}
\end{array}\right]
$$

This tensor is used to relate the orthogonal components of one 3D vector field with those of another, independent vector field. In the example provided in this paper these two fields represent the magnetic fields produced by two coils, with one acting as a transmit coil and another acting as a receive coil. Due to reciprocity the receive coil is simulated in the same way as the transmit coil. In this instance the two vector quantities are combined in a manner which allows for cross-multiplication between dimensions. Consequently, the tensor is capable of weighting complex contributions of a particular object across a six-dimensional field $-\mathrm{H}_{\mathrm{xx}}, \mathrm{H}_{\mathrm{xy}}, \mathrm{H}_{\mathrm{xz}}, \mathrm{H}_{\mathrm{yy}}, \mathrm{H}_{\mathrm{yz}}, \mathrm{H}_{\mathrm{zz}}$. The magnitudes of these contributions are determined by the aspect ratio of an arbitrary $3 \mathrm{D}$ object. The complex nature of the tensor relates to the conductive and magnetic properties of the object. For a magnetic, non-conducting object the tensor components are entirely real, as the object concentrates the primary (incident) magnetic field along its axis. For a conductive, non-magnetic object, the primary field induces eddy currents which circulate within the object, thereby producing a secondary magnetic field. This secondary field is represented in the complex components of the tensor. Similarly, for an object which is both magnetic and conductive, e.g. ferritic steel, the tensor will also contain complex components. Figure 2 below shows some examples of tensors for some special cases. A scalar multiple, $k$, indicates the magnitude of individual tensor components for (a) a sphere, (b) a magnetic rod of negligible diameter with respect to length, (c) a non-magnetic disc of negligible thickness with respect to diameter, and (d) a magnetic disc of negligible thickness with respect to diameter.

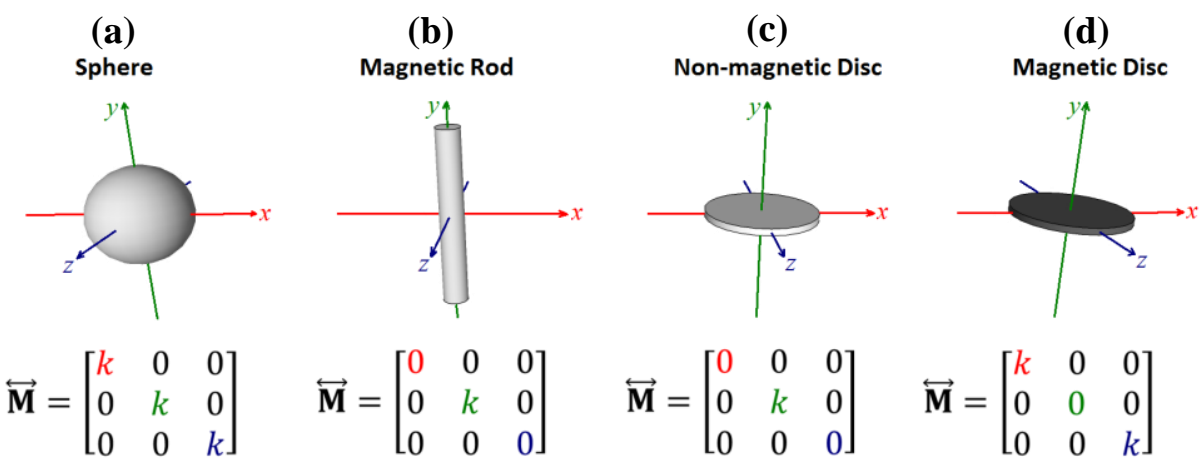

Figure 2. Example of tensors for special case objects.

As a final introductory comment, the tensor contains information relating to object composition and aspect ratio, and therefore can be considered to describe a target object to such an extent as to form the basis of object recognition through the addition of classification. For example a knife would typically consist of a dominant direction (the length of the blade), and smaller dimension (the width of the blade) and a negligible dimension (the thickness of the blade). A further example is that of the barrel of a gun, which would exhibit axial symmetry. As such threat objects tend to have a single dominant dimension it is possible to use the tensor components to verify whether an object tensor represents that of a perceived threat. However, the tensor varies as a function of frequency according to the object's material composition [4], and consequently it is necessary to capture the full spectroscopic contents of the tensor for a more complete representation of the target object. In light of this it is possible to say that recovery of the spectroscopic response of an object may consequently help with the characterisation of the object. Such spectral information is not considered in this paper, and the tensors produced are point values of this spectroscopic information at a single frequency of approximately $12 \mathrm{kHz}$, although clearly the procedures described in this paper could be repeated at several frequencies to obtain a full complex, spectral tensor description of the metallic object. 


\section{Theory}

The derivation of the magnetic polarisability tensor theory is rarely reported in scientific literature and consequently, it has been included below. The theory is presented with the consideration of a system containing two coils - one transmit coil and one receive coil, and the presence of an object at an arbitrary location relative to the coil pair.

\subsection{Practical limitations}

It is possible to invert full Maxwell Equations without any limitations, however would be extremely computationally intensive, and would significantly increase the complexity of the system; given sufficient computational resources and processing time it would be possible to implement such a solution using a finite element (FE) approach. In this instance the Biot-Savart law has been used in order to provide a balance between numerical accuracy and system complexity.

The derivation contains a number of important assumptions which, although not prohibitively restrictive in many cases, it is important to clarify before suitable applications may be considered. The first two of these assumptions are derived from those described in the Biot-Savart law; these being that the conductors are filamentary and exist in vacuo. It is practically impossible to fully satisfy these conditions, however in order to approximate these requirements the conductor bunches used to form each coil have been tightly packed together to minimise unwanted air gaps, and care has been taken to ensure that unnecessary objects are not placed in the vicinity of the coils. It should be noted that although the Biot-Savart law has been used in this paper, largely for computational convenience, other techniques for electromagnetic field calculation, such as the finite element method could be employed.

It is also assumed that the incident (primary) field is parallel across the target object, and that the secondary field (as experienced by the receive coil) can be accurately approximated by the field of a magnetic dipole. To approximately satisfy the requirements for a parallel field, then the maximum object dimension(s) are constrained to be of the order of three times smaller than the distance from the coils. In the context of the detector this corresponds to object sizes of approximately $8 \mathrm{~cm}$. Additionally, a magnetic dipole approximation may be used to represent a current loop, where the loop has dimensions which are much smaller than the distance to the point under observation [5].

\subsection{Derivation of the theory}

The magnetic field produced by the current within a conductor can be calculated at a point relative to the conductor as a function of current, $I$, a linear segment of the conductor, $\mathbf{d} \overrightarrow{\mathbf{L}}$, and the distance between the point of observation and the conductor midpoint $\overrightarrow{\mathbf{r}}$. This relationship is known as BiotSavart law and is shown as equation (1).

$$
\overrightarrow{\mathbf{B}}=\frac{\mu_{0} I}{4 \pi} \oint \frac{\mathbf{d} \overrightarrow{\mathbf{L}} \times \hat{\mathbf{r}}}{r^{2}}
$$

Figure 3 defines a system consisting of a conductor, $\mathbf{d} \overrightarrow{\mathbf{L}}$, and a point of observation, $\overrightarrow{\mathbf{P}}$. The angles $\alpha_{1}$ and $\alpha_{2}$ are defined relative to the Euclidean distance to the line formed by an infinitely long line running along the length $\mathbf{d} \overrightarrow{\mathbf{L}}$, and the distance between $\overrightarrow{\mathbf{P}}$ and the points at both limits of $\mathbf{d} \mathbf{\mathbf { L }}\left(\mathrm{P}_{1}\right.$ and $\mathrm{P}_{2}$ respectively).

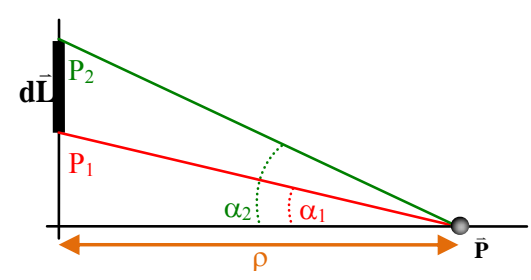

Figure 3. Geometry of parameters for magnetic field calculation. 
It is possible to calculate the magnetic field that is experienced at point $\overrightarrow{\mathbf{P}}$ as a result of current flowing through conductor $\mathbf{d} \overrightarrow{\mathbf{L}}$ by using an adapted version Biot-Savart law as indicated in equation (2) below. In this equation $\hat{\mathbf{a}}_{\mathrm{k}}$ represents a unit vector orthogonal to the plane formed by $\mathbf{d} \mathbf{\mathbf { L }}$ and $\rho$.

$$
\overrightarrow{\mathbf{H}}_{P(x, y, z)}=\frac{I}{4 \pi \rho}\left(\sin \alpha_{2}-\sin \alpha_{1}\right) \hat{\mathbf{a}}_{\mathrm{k}}
$$

Faraday's law states that the source of this magnetic field on an arbitrary, rigid, closed circuit, $C$, in a time-varying magnetic field is also the source of the electric field [6]. This is shown in equation (3), where $\mathbf{E}$ is the electric field strength and $\mathbf{A}$ is the magnetic vector potential. The total EMF induced in this circuit can be calculated by taking the line integral of the electric field around $C$ [7] as shown in equation (4).

$$
\begin{aligned}
\oint_{C} \overrightarrow{\mathbf{E}} \cdot \mathbf{d} \overrightarrow{\mathbf{L}} & =-\oint_{C} \frac{\partial \overrightarrow{\mathbf{A}}}{\partial t} \cdot \mathbf{d} \overrightarrow{\mathbf{L}} \\
V_{\text {ind }} & =\oint_{C} \overrightarrow{\mathbf{E}} \cdot \mathbf{d} \overrightarrow{\mathbf{L}}
\end{aligned}
$$

By integrating $\overrightarrow{\mathbf{A}} \cdot \mathbf{d} \mathbf{\mathbf { L }}$ around $C$ it is possible to calculate the value for flux $\phi$ linking $C$ [8]. This can then be equated to the induced EMF by adding a $j \omega$ term, assuming sinusoidal excitation and adopting standard complex phasor notation, as shown in equation (5).

$$
V_{\text {ind }}=-j \omega \phi=-\oint_{C} j \omega \overrightarrow{\mathbf{A}} \cdot \mathbf{d} \overrightarrow{\mathbf{L}}
$$

Equation (6) shows the leading term expansion for magnetic vector potential for a magnetic dipole moment, $\overrightarrow{\mathbf{m}}[9]$. A magnetic dipole moment is defined as the maximum torque experienced by a magnet when placed in the presence of an external magnetic field [10]. In this case the magnet in question corresponds to the object that is present, and the external field refers to the $\overrightarrow{\mathbf{H}}$-field calculated using (1). The units of magnetic dipole moment are A. $\mathrm{m}^{2}$ [10]. Equation (6) can then be substituted into (5) and the resulting vector operations can be rearranged to produce equation (7). This allows for (1) to be substituted into (7) and consequently replaces a large number of terms on the right hand side with $\overrightarrow{\mathbf{H}}$ as shown in equation (8). The resulting $I$ term in the denominator refers to the induced voltage due to the magnetic dipole, consequently it is necessary to indicate that the current, $I$, and magnetic field, $\overrightarrow{\mathbf{H}}$, are in reference to the receive coil. The subscript ' $R$ ' has been used to indicate this.

$$
\begin{gathered}
\overrightarrow{\mathbf{A}} \approx \frac{\mu_{0}}{4 \pi} \frac{\overrightarrow{\mathbf{m}} \times \hat{\mathbf{r}}}{r^{2}} \\
V_{\text {ind }} \approx \frac{j \omega \mu_{0}}{4 \pi} \frac{\overrightarrow{\mathbf{m}} \cdot(\hat{\mathbf{r}} \times \mathbf{d} \overrightarrow{\mathbf{L}})}{r^{2}} \\
V_{\text {ind }} \approx \frac{j \omega \mu_{0}}{I_{R}} \overrightarrow{\mathbf{m}} \cdot \overrightarrow{\mathbf{H}}_{R}
\end{gathered}
$$

At this point it is possible to introduce a dyadic tensor, which relates the magnetic dipole moment, $\overrightarrow{\mathbf{m}}$ to the field acting upon the object $\overrightarrow{\mathbf{H}}_{T}$ [11]. This quantity is represented as $\overrightarrow{\mathbf{M}}$, and its definition is shown in equation (9). The SI units for $\overrightarrow{\mathbf{M}}$ are $\mathrm{m}^{3}$. Equation (10) is produced by substituting (9) into (8), and indicates a clear dependency upon $V_{\text {ind }}=K \overrightarrow{\mathbf{M}} \cdot \overrightarrow{\mathbf{H}}_{T} \cdot \overrightarrow{\mathbf{H}}_{R}$. 


$$
\begin{gathered}
\overrightarrow{\mathbf{m}}=\overrightarrow{\mathbf{M}} \cdot \overrightarrow{\mathbf{H}}_{T} \\
V_{\text {ind }}=K \overrightarrow{\mathbf{M}} \cdot \overrightarrow{\mathbf{H}}_{T} \cdot \overrightarrow{\mathbf{H}}_{R} \quad \text { where } \quad K=\frac{j \omega \mu_{0}}{I_{R}}
\end{gathered}
$$

As the values of magnetic field can be pre-computed for a particular coil array, it is possible to combine this information to produce a system matrix, $\mathbf{A}_{P}$. The subscript ' $\mathrm{P}$ ' is used to denote the fact that the sensitivity matrix is dependent upon location relative to the coil network. A system measurement, $b$, can be partially expressed as the product of the magnetic polarisability tensor and the system matrix. This is shown in equation (11). This relationship is defined to be the forward model for the system, denoted as $f(\overrightarrow{\mathbf{P}}, \overrightarrow{\mathbf{M}})$.

$$
b=\overrightarrow{\mathbf{M}} \cdot \mathbf{A}_{P}
$$

To account for the effect of $K$ it is necessary to multiply $b$ by a calibration constant in order to represent measurable values.

For the inversion the magnetic field information contained within the system matrix $\mathbf{A}_{P}$ is augmented with positional information to form a new matrix, J. This facilitates the solution of both object tensor, $\overrightarrow{\mathbf{M}}$, and object location, $\overrightarrow{\mathbf{P}}$. The inversion algorithm uses a modified LevenbergMarquardt method [12,13] to find the least squares solution to equation (12). In this equation the quantity $\overrightarrow{\mathbf{V}}$ represents a vector of known sequential measurements, and $f(\overrightarrow{\mathbf{P}}, \overrightarrow{\mathbf{M}})$ represents the results of the forward model as a function of the unknown values of object location and magnetic polarisability tensor. On the first iteration the algorithm performs an estimation of object location based upon analysis of the magnitude of the receive signal. This estimate is updated in $\mathbf{J}$ and the algorithm executes. Each iteration of the algorithm returns an updated set of object locations and the subsequent iteration uses the extracted sensitivity components from $\mathbf{A}$ which corresponds to these locations. This information is then updated in $\mathbf{J}$ and the execution continues.

$$
\arg \min \left(\|\overrightarrow{\mathbf{V}}-f(\overrightarrow{\mathbf{P}}, \overrightarrow{\mathbf{M}})\|^{2}\right)
$$

The inversion algorithm is described in equation (13) below.

$$
[\ddot{\mathbf{M}} \overrightarrow{\mathbf{P}}]=\left(\mathbf{J}^{T} \mathbf{J}+\lambda \mathbf{L}^{T} \mathbf{L}\right)^{-1} \mathbf{J}^{T} \overrightarrow{\mathbf{R}}
$$

In this equation $\lambda$ refers the regularisation parameter and $\mathbf{L}$ refers to a regularisation matrix. The value of $\lambda$ is altered during the iteration of the algorithm according to whether the inverted location is within the detector space. The term $\overrightarrow{\mathbf{R}}$ represents the residual value, as defined in equation (14). The residual value is used to provide an estimate of the relative success of the inversion algorithm, in addition to specifying the stopping criterion for the iteration. The value is monitored on each iteration, and when the residual values begins to increase i.e. the computed solution is getting further from the observed measurements, then the algorithm terminates.

$$
\overrightarrow{\mathbf{R}}=\overrightarrow{\mathbf{V}}-f(\overrightarrow{\mathbf{P}}, \overrightarrow{\mathbf{M}})
$$

\section{Experimental setup}

Three experiments are presented in this paper. These experiments investigate the consistency and accuracy of the inverted location and tensors for a variety of object types. 
A total of 500 measurements have been recorded for each walkthrough scan, each with a sampling resolution of 18-bits. To position the object to within several centimetres of the known location a signal-to-noise ratio greater than $15 \mathrm{~dB}$ is typically required. All of the test objects in this paper meet this criterion given an excitation current of 0.4 A RMS. In order to invert tensor components to the level of accuracy presented in this paper it is also necessary to ensure that the coil array is capable of producing a magnetic field which is sensitive in all directions for both transmitter and receiver, thereby ensuring that the sensitivity matrix has significant components across all six dimensions as described in section 1. If these criteria are met then it will be possible to reconstruct tensors to the accuracy presented in this paper regardless of the number of coils, and their locations. The geometry used in this experiment is shown in figure 4 below:

(a)

Coil Geometry

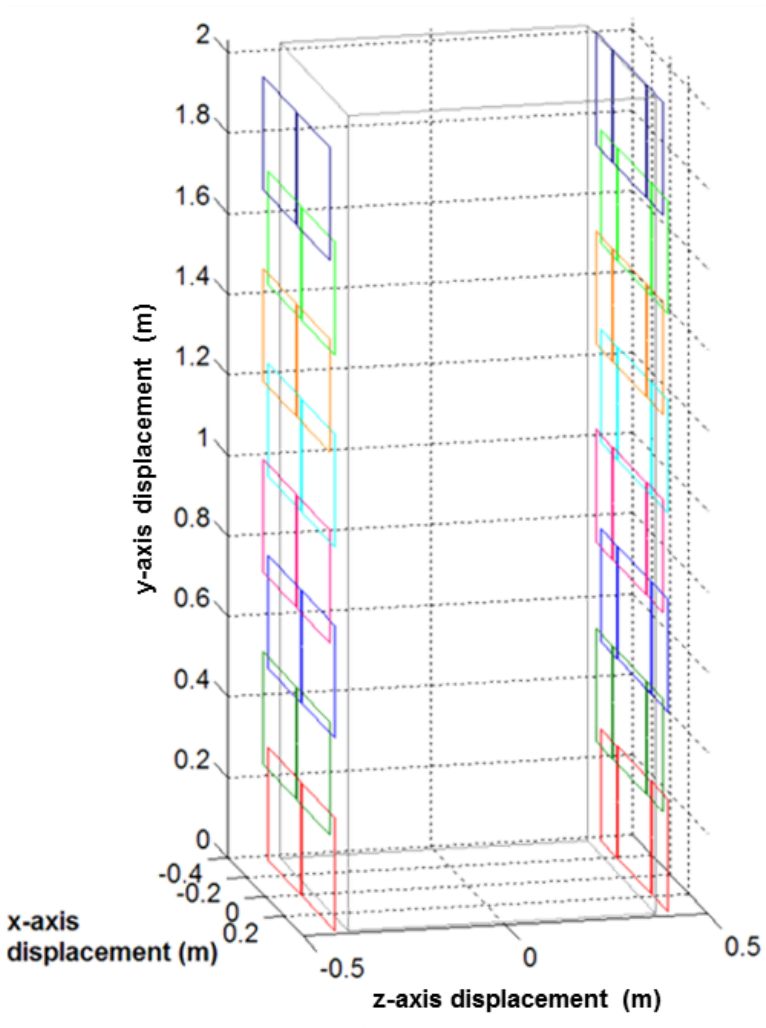

(b)

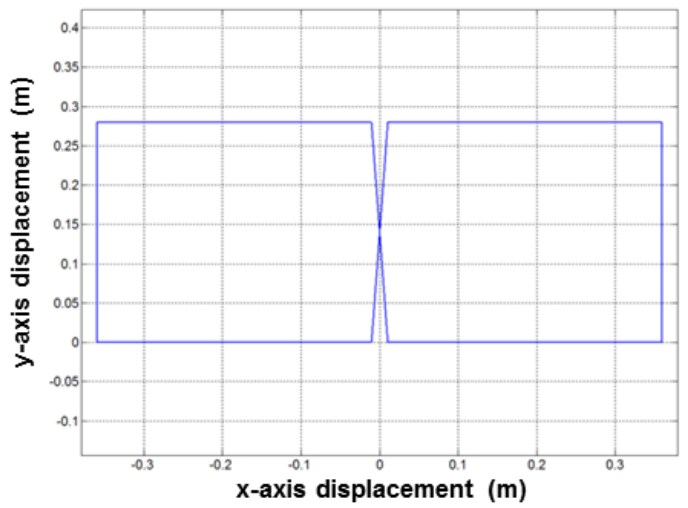

(c)

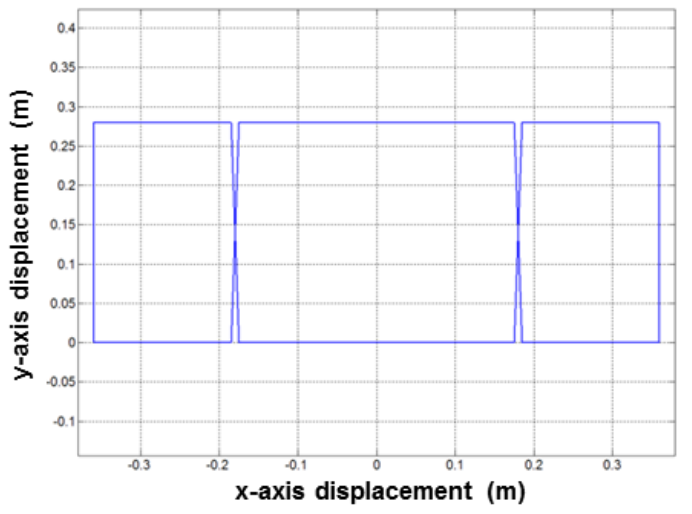

Figure 4. Coil geometry (a) full array, (b) transmit coil design and (c) receive coil design

\subsection{Experiment $1-$ multiple object testing at fixed orientations}

In this experiment, a total of four different objects are reported; these objects are shown below in figure 5, on the background of a cm grid. The object set can be seen to consist of a ferrite sphere, a ferrite rod, an aluminium National Institute of Justice (NIJ) handgun [14], and a steel NIJ handgun. The National Institute of Justice is an agency within the US Department of Justice, and they produce the standard test objects for which US metal detectors are tested against [14]. These test objects are designed to mimic the approximate dimensions and bulk material composition of real threat objects using innocuous materials. These objects have been presented in this paper as they represent a wide range of object tensors. Some details of the expected tensors are shown overleaf. 

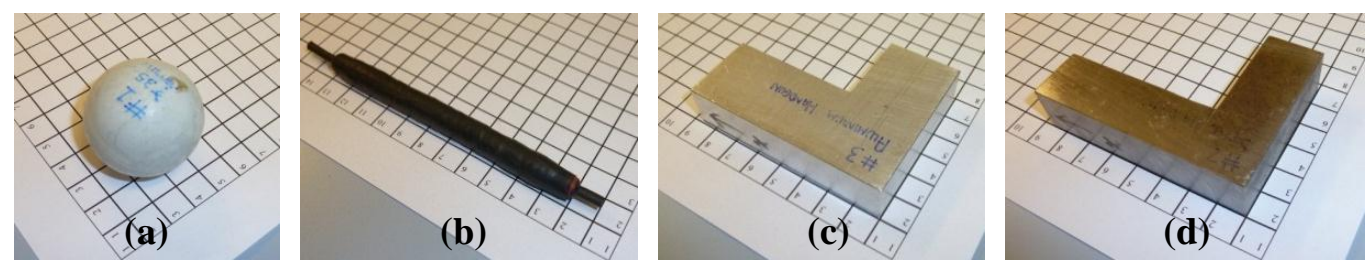

Figure 5. Test objects (a) ferrite sphere, (b) ferrite rod, (c) aluminium NIJ handgun and (d) Steel NIJ handgun.

The sphere has uniform contributions in all directions. The expected tensor consists of values in the diagonal components only i.e. the ' $x x$ ', 'yy' and ' $z z$ ' components. The object is filled with ferrite powder, so that it is magnetic and non-conductive. In order to reflect this the tensor should consist of real parts only.

The rod has very small cross-sectional dimensions compared to its length. It is composed from ferrite beads that have been glued using a non-conductive adhesive. Consequently, as with the sphere, it is magnetic and non-conductive, and should yield a real tensor. When the rod is aligned with one of the principal axes (with respect to the coils) it is expected that the dominant tensor value will be the diagonal component which corresponds to this direction, i.e. $M_{11}$ when aligned with the x-direction, $M_{22}$ when aligned with the y-direction, and $M_{33}$ when aligned with the z-direction.

The tensors for the NIJ guns are expected to have very different values according to both the alignment of the object, and the nature of the conductive and/or magnetic nature of the object. Aluminium has a high conductivity and is non-magnetic, and consequently it is expected to contain negative values by virtue of the fact that the secondary field produced seeks to oppose that of the primary. Additionally, it is not expected that the dominant populated components will lie in the same axis as the preferential direction of the object as the ferrite rod should. This is due to the manner in which conductive objects respond to the incident field. As discussed previously, the primary field induces circulating eddy currents; as these currents circulate they generate their own magnetic field, orthogonal to the plane in which they are moving. Consequently it is expected that the largest tensor component will correspond to the axis orthogonal to the plane containing the largest cross-sectional area of the material. In contrast to this, steel is a combination of conductive metals and, due to its iron content, it is also magnetic. Consequently it is expected that the resulting tensor should have both real and imaginary parts, and the real parts could contain any conceivable value, which would be determined by its conductive and magnetic make-up.

Each scan was conducted with the dominant component aligned with the $\mathrm{x}$-axis as shown in figure 6.
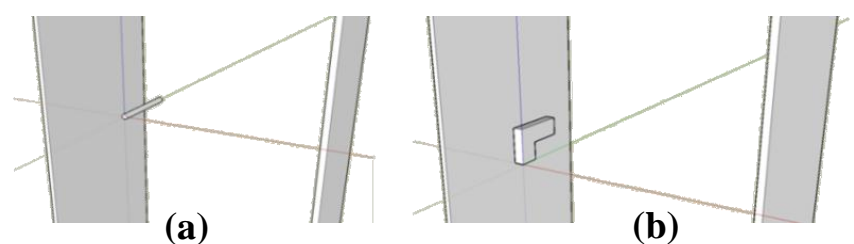

Figure 6. Reference orientations for (a) ferrite rod and (b) NIJ handguns

Each object was carried through the detector spread along a number of different trajectories as shown in figure 7(a). The sphere was scanned five times at each of the five trajectories shown - a total of 25 scans. The other objects were scanned 15 times at the trajectories labelled 'Left Shoulder', 'Waist' and 'Right Pocket' in figure 7(a). Markers were positioned on the floor to allow for a consistent walk-through profile, and a laser measuring tool with a precision of $\pm 1 \mathrm{~mm}$ was used to initially position the test objects. During the walk-through the objects were kept as close as possible to a constant position and orientation. It is assumed that the objects will not have moved by more than 2 or $3 \mathrm{~cm}$ in the $y$ or $z$-direction during the walk-through. 


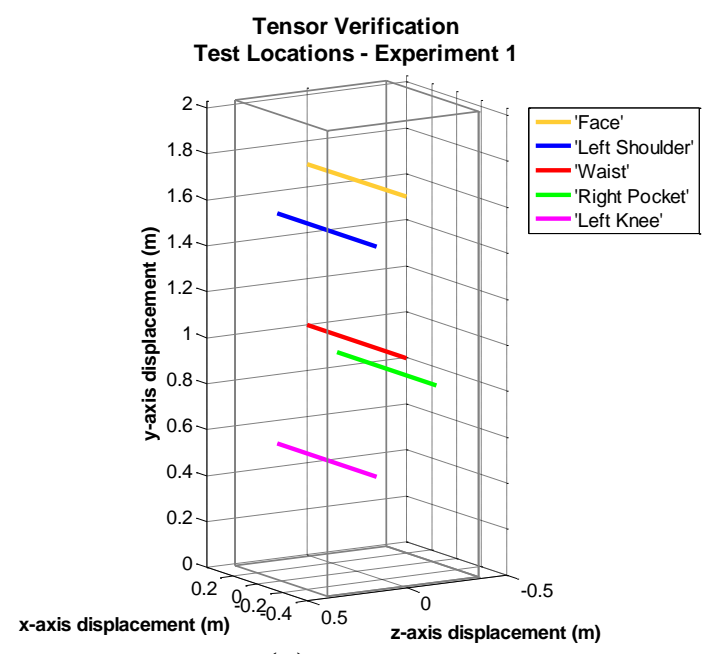

(a)

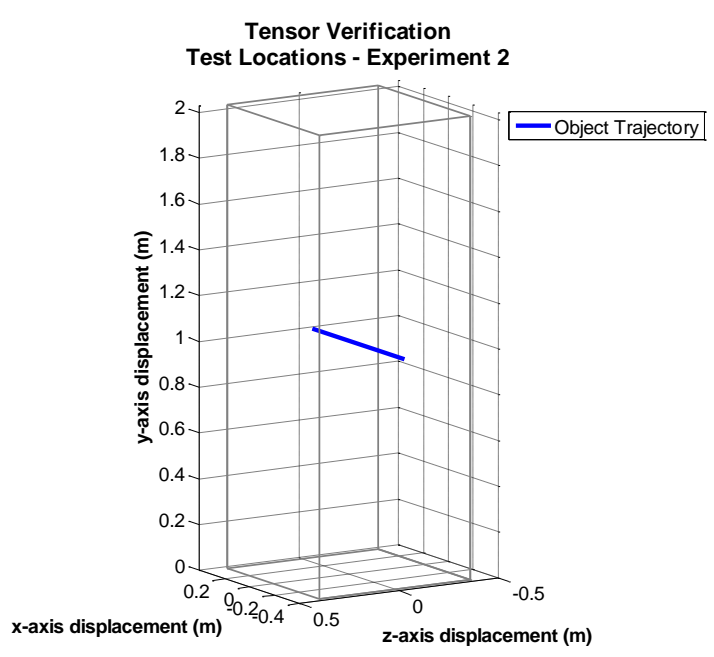

(b)

Figure 7. Trajectories for testing of inversion algorithm (a) for experiment 1, (b) for experiment 2

3.2. Experiment 2 - comparison of results for objects both aligned and not aligned with principle axes In this experiment the steel gun shown in figure 5(d) was carried at the trajectory shown in figure 7(b). A total of 40 walk-through scans were conducted at each of the orientations shown in figure 8 below.

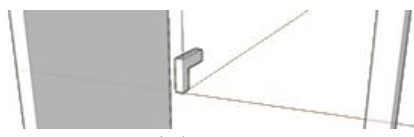

(a)

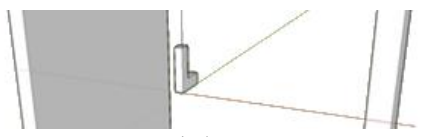

(b)

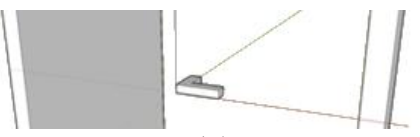

(c)

Figure 8. Scan orientations (a) barrel aligned with $x$-axis, (b) barrel aligned with y-axis and (c) barrel aligned with z-axis.

Four different rotations were then applied so that the object was no longer aligned along the three principal axes. These rotations are described below and shown on figure 9. At each orientation 15 walk-through scans were performed.

(a) The initial ' $x$ ' orientation for each object in figure $7(a)$ rotated $45^{\circ}$ clockwise about the $z$-axis.

(b) The initial ' $y$ ' orientation for each object in figure 7 (b) rotated $45^{\circ}$ counter-clockwise about the $x$-axis.

(c) The initial ' $z$ ' orientation for each object in figure 7(c) rotated $45^{\circ}$ clockwise about the $y$-axis.

(d) Rotation (c) with a further rotation of $45^{\circ}$ counter-clockwise about the $x$-axis.

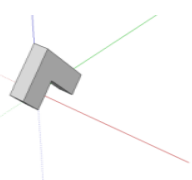

(a)

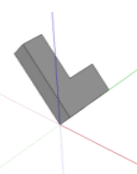

(b)

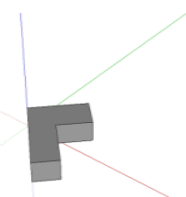

(c)

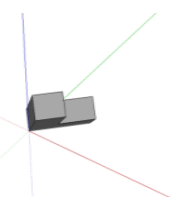

(d)

Figure 9. Object rotations not aligned with the principle axes

The objects were attached to a block of wood, on which angles had been measured using a protractor with an estimated degree of accuracy of $0.5^{\circ}$. A test conducted using a laser spirit level indicated that the error in angle caused due to the walk-through action was rarely greater than $10^{\circ}$, with a more typical value of $<5^{\circ}$. The inverted tensors were rotated using equation (15) below, to align 
the angular offsets in figure 9 so that the orientations correspond to those shown in figure 8 . This allows for direct comparison of the two sets of results. In (15) the values $\hat{\mathbf{M}}$ and $\overrightarrow{\mathbf{M}}$ represent the unrotated and rotated tensors respectively.

$$
\overrightarrow{\mathbf{M}}_{(i)}=\sum_{j=1}^{3} \mathbf{a}_{(i, j)} \hat{\mathbf{M}}_{(j)} \quad i=1,2,3
$$

The value a in (15) represents the dyadic rotation matrix [15] as shown in equation (16). In this equation the angles $\theta_{x}, \theta_{y}$ and $\theta_{z}$ correspond to a rotation about the $\mathrm{x}, \mathrm{y}$ and zaxes respectively.

$$
\begin{aligned}
& \mathbf{a}\left(\theta_{x}, \theta_{y}, \theta_{z}\right)= \\
& {\left[\begin{array}{ccc}
\cos \left(\theta_{z}\right) \cos \left(\theta_{y}\right)-\sin \left(\theta_{z}\right) \sin \left(\theta_{x}\right) \sin \left(\theta_{y}\right) & \sin \left(\theta_{z}\right) \cos \left(\theta_{y}\right)+\cos \left(\theta_{z}\right) \sin \left(\theta_{x}\right) \sin \left(\theta_{y}\right) & -\cos \left(\theta_{x}\right) \sin \left(\theta_{y}\right) \\
-\sin \left(\theta_{z}\right) \cos \left(\theta_{x}\right) & \cos \left(\theta_{z}\right) \cos \left(\theta_{x}\right) & \sin \left(\theta_{x}\right) \\
\cos \left(\theta_{z}\right) \sin \left(\theta_{y}\right)+\sin \left(\theta_{z}\right) \sin \left(\theta_{x}\right) \cos \left(\theta_{y}\right) & \sin \left(\theta_{z}\right) \sin \left(\theta_{y}\right)-\cos \left(\theta_{z}\right) \sin \left(\theta_{x}\right) \cos \left(\theta_{y}\right) & \cos \left(\theta_{x}\right) \cos \left(\theta_{y}\right)
\end{array}\right]}
\end{aligned}
$$

\subsection{Experiment 3 -object location for a controlled trajectory}

This experiment demonstrates the accuracy of the detector when inverting the location of a threat object. A sphere (of diameter $37 \mathrm{~mm}$ ) filled with nickel zinc ferrite powder was used as the test object (see figure 5(a)). A total of five different trajectories were used at various locations within the detector, which are shown in figure 10. At each location a total of 20 scans were conducted, and the inverted locations were stored. For each trajectory all inverted locations are plotted on the same figure, along with the actual position of the test object.

In all cases the ball was rolled through the detector on a fixed plastic rail. This rail allowed the ball to travel through all $\mathrm{x}$-values of the detector at a constant $\mathrm{y}-\mathrm{z}$ location. The rail was positioned using a laser distance measuring tool which has an accuracy of $\pm 1 \mathrm{~mm}$. Allowing for a small level of vibration there is the possibility of a further uncertainty of approximately $\pm 1 \mathrm{~mm}$; trajectories labelled as 'known location' can be assumed to have this level of accuracy.
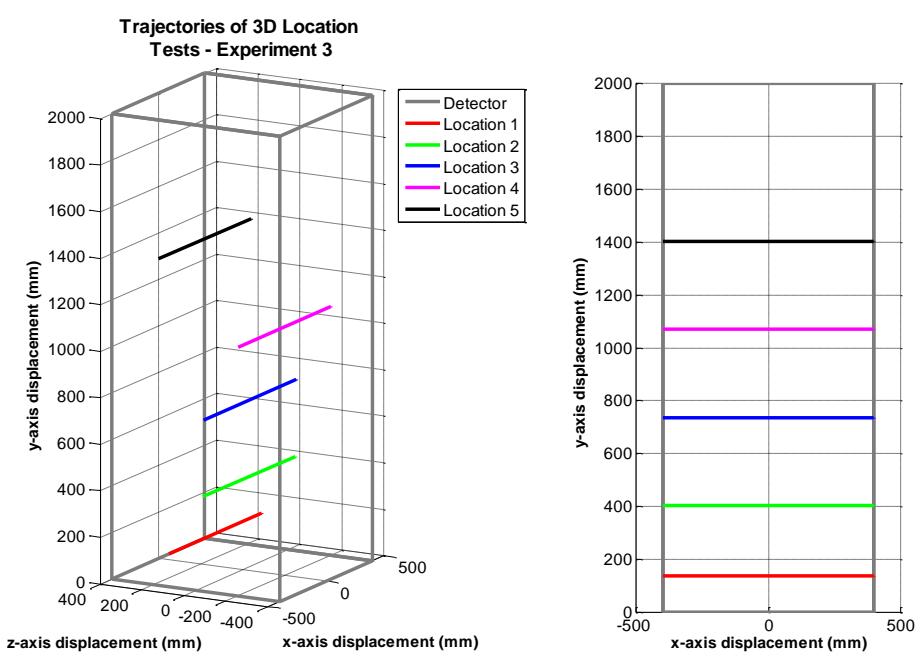

Figure 10. Controlled trajectories

\subsection{Presentation of inverted tensors}

All of the tensors presented in the results section have been averaged from the full set of inverted tensors. In the case of experiment 1 the average tensor is calculated per object, and for experiment 2 the average tensor is calculated per orientation. 


\section{Results}

\subsection{Experiment 1}

The results for the testing using the object displayed in figure 5 (a) - (d) are as follows:

(a) Ferrite sphere

$$
\overrightarrow{\mathbf{M}}=\left[\begin{array}{ccc}
0.9444-0.0144 \mathrm{j} & -0.0153-0.0076 \mathrm{j} & 0.0284+0.0107 \mathrm{j} \\
-0.0153-0.0076 \mathrm{j} & 0.812+0.0445 \mathrm{j} & 0.0152-0.0092 \mathrm{j} \\
0.0284+0.0107 \mathrm{j} & 0.0152-0.0092 \mathrm{j} & 0.8472-0.0271 \mathrm{j}
\end{array}\right]
$$

Note that this tensor is as close to what is expected for an object of this type, i.e. the unity matrix. The magnetic, non-conductive nature of the object is shown in the fact that the dominant components are real values, and the similarity in the diagonal components implies a comparable aspect ratio in all dimensions i.e. a sphere. It is possible to see that these components are not equal, as would be expected for an ideal spherical case. However, the values are sufficiently close to one another to determine that this object has approximately equal contributions in the three orthogonal components.

(b) Ferrite rod

$$
\overrightarrow{\mathbf{M}}=\left[\begin{array}{ccc}
1.6084-0.0118 \mathrm{j} & -0.1195+0.0003 \mathrm{j} & 0.1317-0.0019 \mathrm{j} \\
-0.1195+0.0003 \mathrm{j} & 0.1816+0.0126 \mathrm{j} & 0.0195+0.0069 \mathrm{j} \\
0.1317+0.0019 \mathrm{j} & 0.0195+0.0069 \mathrm{j} & 0.2162-0.0496 \mathrm{j}
\end{array}\right]
$$

This tensor shows a dominant value in element $M_{11}$, which corresponds to the 'xx' component of the tensor. This corresponds to the orientation of the rod as shown in figure 6(a), as the preferential material direction of the rod is aligned along the $\mathrm{x}$-axis of both coil geometries. The magnitudes of the two other diagonal components are sufficiently close to one another to infer that the cross-sectional area of the rod is approximately equal with respect to both directions - which is known to be true for the rod. In addition, as with the sphere, it is possible to see that the dominant tensor values are all real; this corresponds to a magnetic and non-conductive rod.

(c) Aluminium gun

$$
\overrightarrow{\mathbf{M}}=\left[\begin{array}{ccc}
-1.2514-0.1257 \mathrm{j} & -0.0272-0.0085 \mathrm{j} & 0.0662+0.0051 \mathrm{j} \\
-0.0272-0.0085 \mathrm{j} & -1.4318-0.1288 \mathrm{j} & -0.0457+0.0088 \mathrm{j} \\
0.0662+0.0051 \mathrm{j} & -0.0457+0.0088 \mathrm{j} & -2.266-0.186 \mathrm{j}
\end{array}\right]
$$

This result shows that the tensor for a conductive object is very different to that of a purely magnetic object, as shown for the ferrite sphere and rod above. The dominant components of this tensor are all negative, real values, as described in section 3 of this paper. The magnitude of these values reflects the fact that the cross-sectional area of the gun is largest in the $x-y$ plane, and hence produces a large ' $z \mathrm{z}$ ' value (element $M_{33}$ in the tensor). The cross-sectional area in the $\mathrm{x}-\mathrm{z}$ and $\mathrm{y}-\mathrm{z}$ planes are both much smaller, and the two values are approximately equal, with the x-z plane being the larger of the two; this is reflected in the slightly larger 'yy' value (element $M_{22}$ in the tensor).

(d) Steel gun

$$
\overrightarrow{\mathbf{M}}=\left[\begin{array}{ccc}
0.8584-1.4056 \mathrm{j} & 0.0065-0.0026 \mathrm{j} & 0.0204-0.0227 \mathrm{j} \\
0.0065-0.0026 \mathrm{j} & 0.743-1.1386 \mathrm{j} & -0.0408+0.0143 \mathrm{j} \\
0.0204-0.0227 \mathrm{j} & -0.0408+0.0143 \mathrm{j} & 0.4257-0.8105 \mathrm{j}
\end{array}\right]
$$


It is possible to see that this is the only tensor in the object set which contains significant imaginary values. As discussed previously, this can be attributed to the variety of magnetic and conductive materials used to produce the steel for this item. The comparison between real and imaginary contributions from the object is one of the properties contained within the tensor which may be used to classify an item. When the magnitude of each tensor element is calculated it is possible to determine that the largest value is contained in the 'xx' component (element $M_{11}$ in the tensor), the second largest value is in the 'yy' component (element $M_{22}$ in the tensor), and the smallest value is in the ' $\mathrm{zz}$ ' component (element $M_{33}$ in the tensor). These correspond with the largest preferential direction of the gun (the barrel) being aligned along the $\mathrm{x}$-axis; the second largest direction (the handle), being aligned in the $y$-direction, and the smallest direction being aligned in the z-direction.

\subsection{Experiment 2}

The inverted locations for all 40 walk-through scans at the orientations shown in figure 8 are shown in figure 11 .

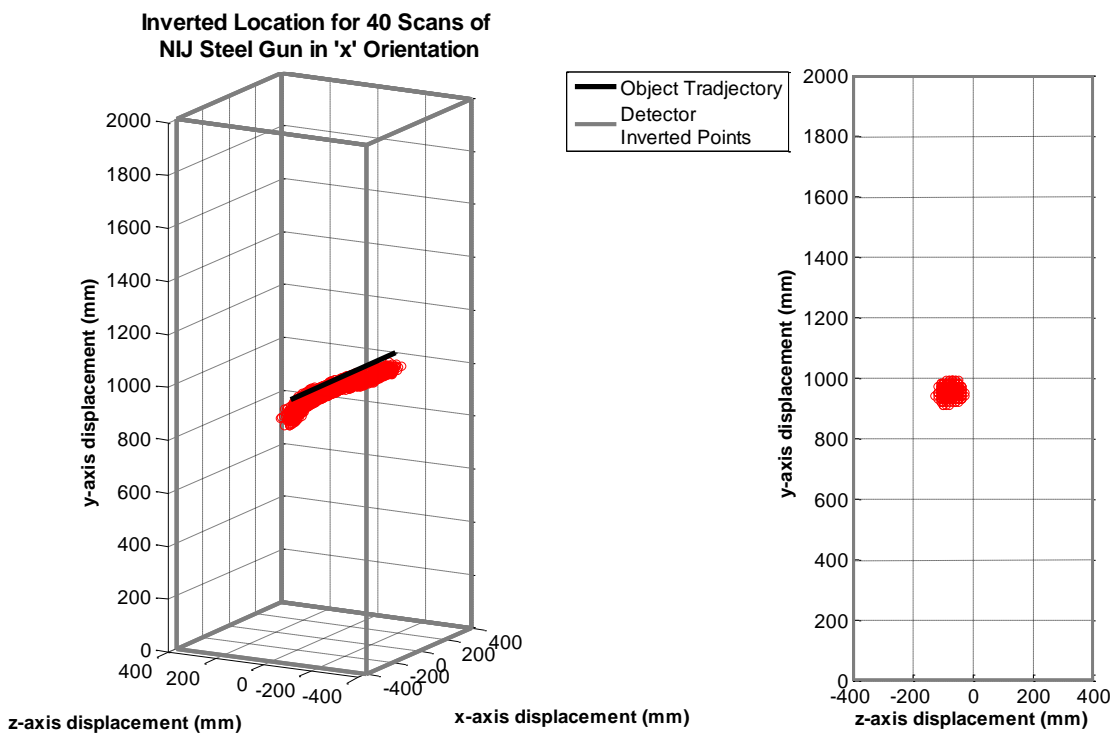

Figure 11. Inverted positional information for all walk-through scans as shown in figure 8

This figure shows that all the inverted locations lie approximately in the same region in the $\mathrm{x}-\mathrm{z}$ plane, and follow a linear trajectory in the x-axis. This correctly reflects the walkthrough profile, and is an encouraging result.

The averaged inverted tensor for the orientation indicated in figure 8(a) is shown below:

$$
\overrightarrow{\mathbf{M}}_{f i g(a)}=\left[\begin{array}{ccc}
0.9000-1.4437 \mathrm{j} & 0.0566+0.0733 \mathrm{j} & 0.0355-0.0264 \mathrm{j} \\
0.0566+0.0733 \mathrm{j} & 0.7139-1.3510 \mathrm{j} & -0.1768-0.0062 \mathrm{j} \\
0.0355-0.0264 \mathrm{j} & -0.1768-0.0062 \mathrm{j} & 0.3465-0.7055 \mathrm{j}
\end{array}\right]
$$

In this instance the gun is in the same orientation as for the testing in 'Experiment 1', consequently the results are directly comparable. When examining both tensors it is possible to see that the values follow similar trends in terms of the sign of the dominant components, and the relative magnitudes with respect to the principal directions of the object. The numerical differences are shown below in table 1. This table shows that the difference in the largest component (corresponding to the preferential direction of the gun) is $4.62 \%$ and $2.64 \%$ for real and imaginary parts respectively. The differences for the remaining values are larger, typically in the region of $15 \%$ to $23 \%$. However, it should be noted 
that the largest value of nearly $23 \%$ corresponds to a considerably smaller value than for the other diagonal components.

Table 1. Differences between averaged tensors for experiments 1 and 2.

\begin{tabular}{cccccc}
\hline \multicolumn{2}{c}{$\mathbf{x x}$} & \multicolumn{2}{c}{$\mathbf{y y}$} & \multicolumn{2}{c}{$\mathbf{z z}$} \\
$\operatorname{Re}\{\mathrm{xx}\}$ & $\operatorname{Im}\{\mathrm{xx}\}$ & $\operatorname{Re}\{\mathrm{yy}\}$ & $\operatorname{Im}\{\mathrm{yy}\}$ & $\operatorname{Re}\{\mathrm{zz}\}$ & $\operatorname{Im}\{\mathrm{zz}\}$ \\
\hline $4.62 \%$ & $2.64 \%$ & $4.08 \%$ & $15.72 \%$ & $22.86 \%$ & $14.88 \%$ \\
\hline
\end{tabular}

Figure 12 displays a plot of the range of inverted tensor components for each of the 40 walkthrough tests. It is possible to see that for each orientation shown in figure 8 the spread of points is typically fairly small, and that all values have an even distribution within the overall range. It should be noted that the spread of tensor values is affected with rotation of the object, and a small degree of rotation (previously defined as $\sim 5^{\circ}$ ) is within the limits of experimental uncertainty. The $\mathrm{x}$-axis is scaled with integer values which correspond to tensor elements in the following way:

$$
\left[\begin{array}{lll}
M_{11} & M_{12} & M_{13} \\
M_{12} & M_{22} & M_{23} \\
M_{13} & M_{23} & M_{33}
\end{array}\right] \quad \Rightarrow \quad\left[\begin{array}{lll}
1 & 2 & 3 \\
2 & 4 & 5 \\
3 & 5 & 6
\end{array}\right]
$$
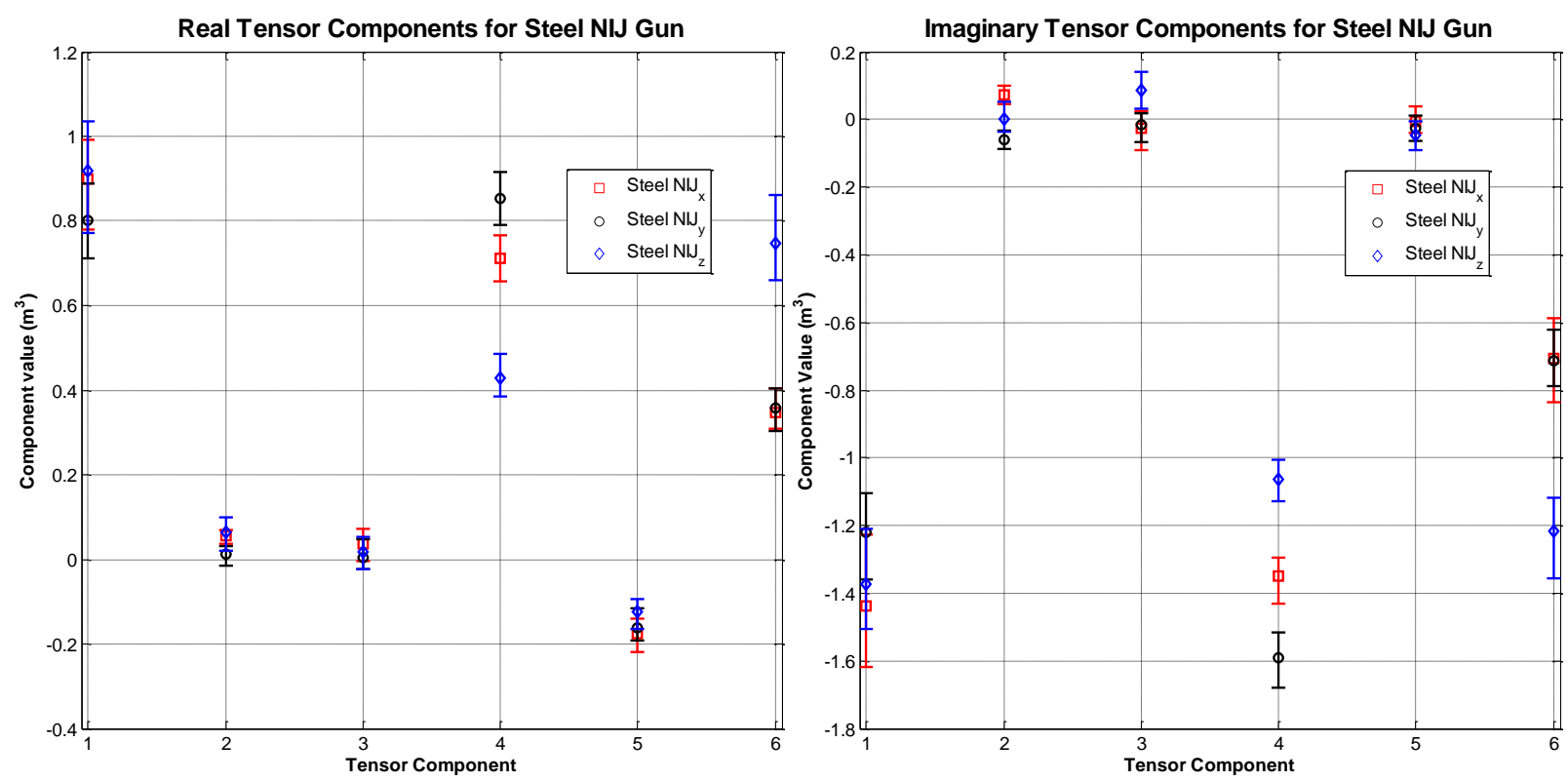

Figure 12. Inverted tensor components for walk-through scans as shown in figure 8

The averaged inverted tensor for all 40 walk-through scans at the orientation shown in figure 8(b) is shown overleaf.

$$
\overrightarrow{\mathbf{M}}_{f i g(b)}=\left[\begin{array}{ccc}
0.8015-1.2259 \mathrm{j} & 0.0129-0.0596 \mathrm{j} & 0.0061-0.0187 \mathrm{j} \\
0.0129-0.0596 \mathrm{j} & 0.8548-1.5924 \mathrm{j} & -0.1606-0.0283 \mathrm{j} \\
0.0061-0.0187 \mathrm{j} & -0.1606-0.0283 \mathrm{j} & 0.3589-0.7107 \mathrm{j}
\end{array}\right]
$$

By transposing the first two diagonal components it is possible to mimic the effect of rotating the object from the previous rotation (with respect to the orthogonal field components). This allows for 
some comparison of the two tensors. Upon comparison of the two tensors it is possible to see that the characteristics of the tensor values correspond to expected values.

The averaged inverted tensor for all 40 walk-through scans at the orientation shown in figure 8(c) is shown below. It is possible to see that the relative magnitudes of the tensor components, as well as their signs, show consistency with the previous orientations.

$$
\overrightarrow{\mathbf{M}}_{f i g(c)}=\left[\begin{array}{ccc}
0.7469-1.2161 \mathrm{j} & 0.0621-0.0025 \mathrm{j} & 0.0171+0.0841 \mathrm{j} \\
0.0621-0.0025 \mathrm{j} & 0.4282-1.0632 \mathrm{j} & -0.1233-0.0484 \mathrm{j} \\
0.0171-0.0841 \mathrm{j} & -0.1233-0.0484 \mathrm{j} & 0.9184-1.3753 \mathrm{j}
\end{array}\right]
$$

For the orientation shown in figure 9(a) the following tensor was produced:

$$
\overrightarrow{\mathbf{M}}_{f i g(a)}=\left[\begin{array}{ccc}
0.7598-1.2456 \mathrm{j} & 0.0886-0.1019 \mathrm{j} & 0.0355+0.0044 \mathrm{j} \\
0.0886-0.1019 \mathrm{j} & 0.7631-1.4643 \mathrm{j} & -0.0920+0.0074 \mathrm{j} \\
0.0355+0.0044 \mathrm{j} & -0.0920+0.0074 \mathrm{j} & 0.3193-0.6522 \mathrm{j}
\end{array}\right]
$$

This tensor was then rotated using equations (15) and (16) to produce the following equivalent:

$$
\overrightarrow{\mathbf{M}}_{\text {fig } 9(a) \text { rotated }}=\left[\begin{array}{ccc}
0.8501-1.4568 \mathrm{j} & 0.0017-0.1094 \mathrm{j} & -0.0399+0.0083 \mathrm{j} \\
0.0017-0.1094 \mathrm{j} & 0.6728-1.2531 \mathrm{j} & -0.0902+0.0021 \mathrm{j} \\
-0.0399+0.0083 \mathrm{j} & -0.0902+0.0021 \mathrm{j} & 0.3193-0.6522 \mathrm{j}
\end{array}\right]
$$

Table 2 shows the error between the tensor components following this rotation, and that the error from the un-rotated orientation shown in figure 8(a). These values are analogous to those experienced as a result of testing at the other orientations indicated in figure 9. Note that tensor components smaller than 0.1 have been omitted for clarity. This shows an agreement of results of typically $<8 \%$ for the

\begin{tabular}{|c|c|c|c|c|c|c|}
\hline & \multicolumn{2}{|c|}{$\mathbf{x}$} & \multicolumn{2}{|c|}{$\mathbf{y}$} & \multicolumn{2}{|c|}{$\mathbf{Z}$} \\
\hline & $\mathrm{Re}$ & Im & $\mathrm{Re}$ & Im & $\mathrm{Re}$ & $\mathrm{Im}$ \\
\hline $\mathbf{x}$ & $5.55 \%$ & $0.91 \%$ & - & - & - & - \\
\hline $\mathbf{y}$ & - & - & $5.75 \%$ & $7.25 \%$ & - & - \\
\hline $\mathbf{Z}$ & - & - & - & - & $7.85 \%$ & $7.55 \%$ \\
\hline
\end{tabular}
dominant tensor components.

Table 2. Difference between tensors for aligned and rotated tests.

For the orientations shown in figure $9(\mathrm{~b})-9(\mathrm{~d})$ the following rotated equivalents tensors were produced:

$$
\begin{aligned}
\overrightarrow{\mathbf{M}}_{\text {fig } 9(b) \text { rotated }} & =\left[\begin{array}{ccc}
0.6934-0.9999 \mathrm{j} & 0.0139-0.0269 \mathrm{j} & 0.0154-0.0425 \mathrm{j} \\
0.0139-0.0269 \mathrm{j} & 0.6605-1.5234 \mathrm{j} & 0.0144+0.1831 \mathrm{j} \\
0.0154-0.0425 \mathrm{j} & 0.0144+0.1831 \mathrm{j} & 0.4030-0.8572 \mathrm{j}
\end{array}\right] \\
\overrightarrow{\mathbf{M}}_{\text {fig } 9(c) \text { rotated }} & =\left[\begin{array}{ccc}
0.6880-1.0144 \mathrm{j} & -0.0615+0.0144 \mathrm{j} & 0.0137+0.0546 \mathrm{j} \\
-0.0615+0.0144 \mathrm{j} & 0.3444-1.0569 \mathrm{j} & -0.0875-0.0044 \mathrm{j} \\
0.0137+0.0546 \mathrm{j} & -0.0875-0.0044 \mathrm{j} & 0.7361-1.2048 \mathrm{j}
\end{array}\right] \\
\overrightarrow{\mathbf{M}}_{\text {fig } 9(d) \text { rotated }} & =\left[\begin{array}{ccc}
0.6620-1.0112 \mathrm{j} & 0.1447+0.0086 \mathrm{j} & 0.1186-0.1458 \mathrm{j} \\
0.1447+0.0086 \mathrm{j} & 0.3164-0.9811 \mathrm{j} & -0.0796+0.2405 \mathrm{j} \\
0.1186-0.1458 \mathrm{j} & -0.0796+0.2405 \mathrm{j} & 0.7796-1.2650 \mathrm{j}
\end{array}\right]
\end{aligned}
$$




\subsection{Experiment 3}

The results for all 20 scans at each of the five trajectories shown in figure 9 are shown on figure 13 . Due to the scale of the figure it is not possible to accurately indicate the region of uncertainty and as a result of this the width of the line is a purely arbitrary choice.

Figure 14 shows the total error across all five trajectories. In each case the known trajectory has been subtracted from the inverted trajectory, hence referring each inverted location to a true value of $(0,0)$ in the $y-z$ plane.

These results show that the system is capable of consistently and accurately inverting object locations with an error of no greater than \pm 10 to $15 \mathrm{~mm}$ in the y-direction, and $\pm 30 \mathrm{~mm}$ in the z-direction. This demonstrates the potential to significantly reduce the region of uncertainty of object location.

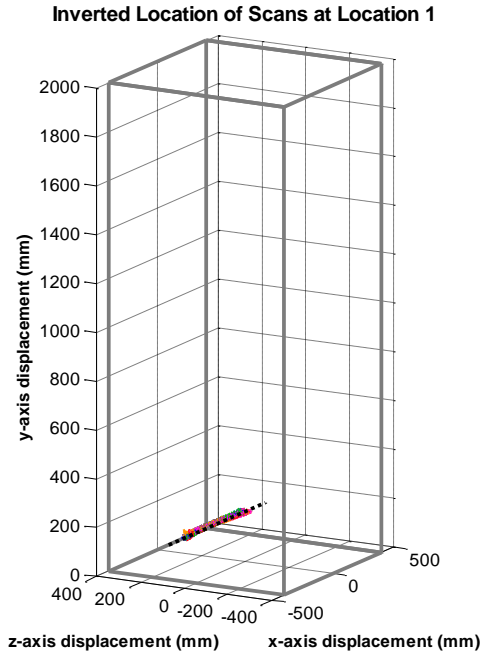

(a)

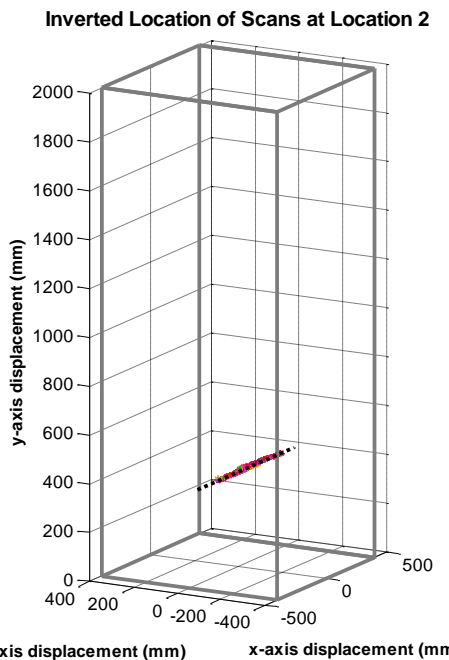

(b)

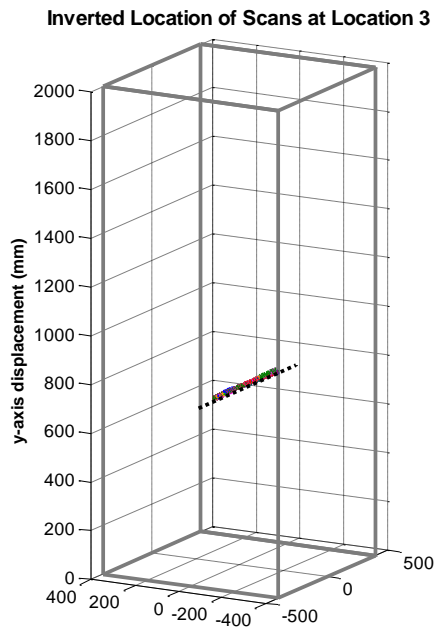

(c)

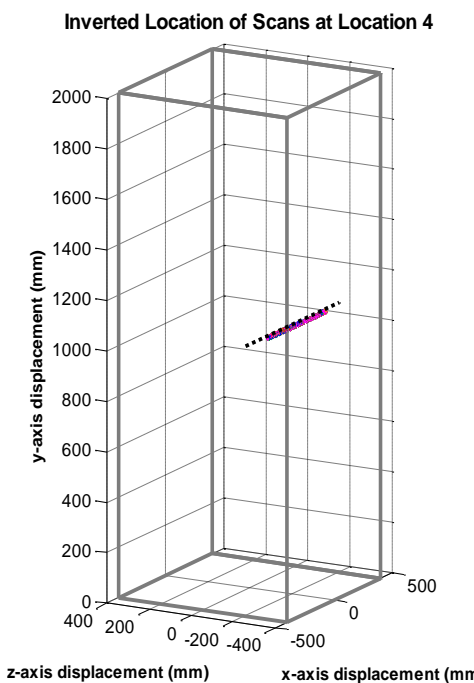

(d)

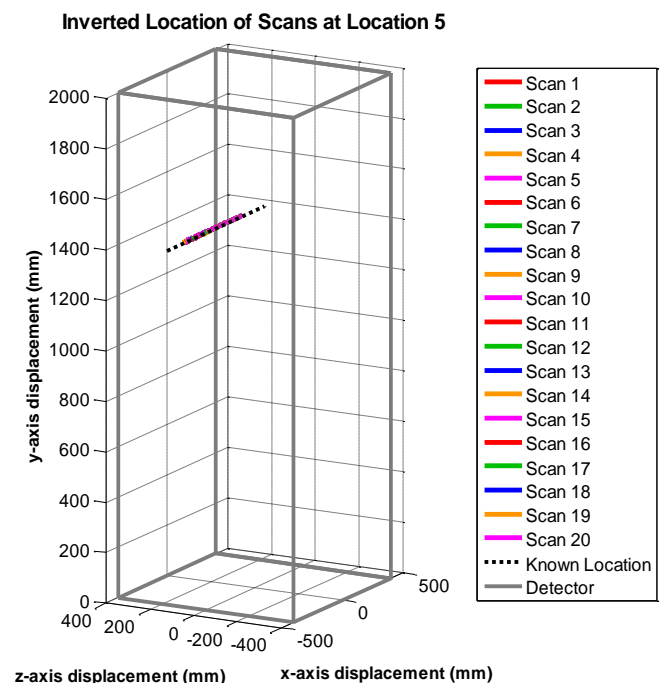

(e)

Figure 13. Inverted locations for experiment 3 
(a)

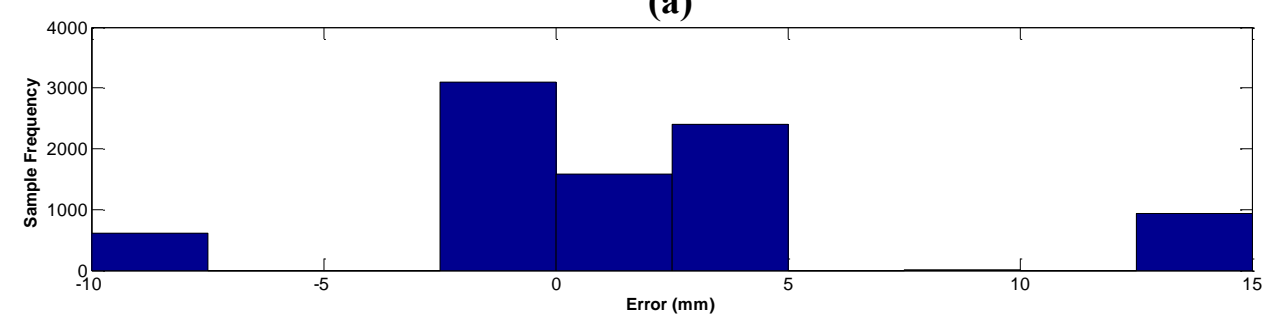

(b)

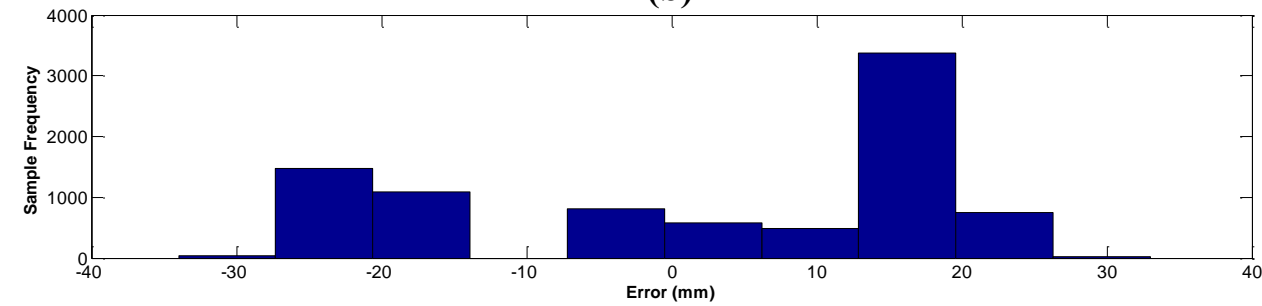

Figure 14. Error in inverted location for (a) y-location, (b) z-location and (c) in the y-z plane

\section{Conclusion}

The results presented in this paper show that it is possible to consistently reconstruct the magnetic polarisability tensor from a single walk-through scan. This has also been shown to be the case for instances where objects are rotated at arbitrary angles, which is a common scenario. The quality of the tensors recovered is such that it is possible to correctly determine the dominance of orthogonal components, and consequently meets the aim of being able to provide the operator with information regarding the likelihood of an object being considered as a threat based on its shape. The ability to distinguish material composition has been introduced by examination of the ratio between real and imaginary tensor components, however to provide the operator with the greatest amount of information concerning material composition it is necessary to perform broadband analysis of the tensor.

In summary it can be said that based on this single frequency system it would be possible to classify an object based on aspect ratio irrespective of location and orientation, assuming a signal-tonoise ratio in excess of $15 \mathrm{~dB}$. However, in order to classify a wide range of objects by material type it is necessary to evaluate the tensor across a wider range of frequencies, as the fixed frequency approach does not guarantee caption of the dominant tensor response with respect to frequency.

The reliability in inverting positional information for a detectable object has also been shown to yield consistent and accurate results, indicating that an object location can be determined to within a matter of centimetres of the true position. This high degree of accuracy potentially allows for walkthrough metal detector operators to carry out a quick, highly constrained physical search, rather than the time-consuming, wide-scale search that the current generation of detectors require. Figure 15 shows how this detailed positional information may be coupled with video technology to provide an image of the candidate with an overlay showing the target location. 


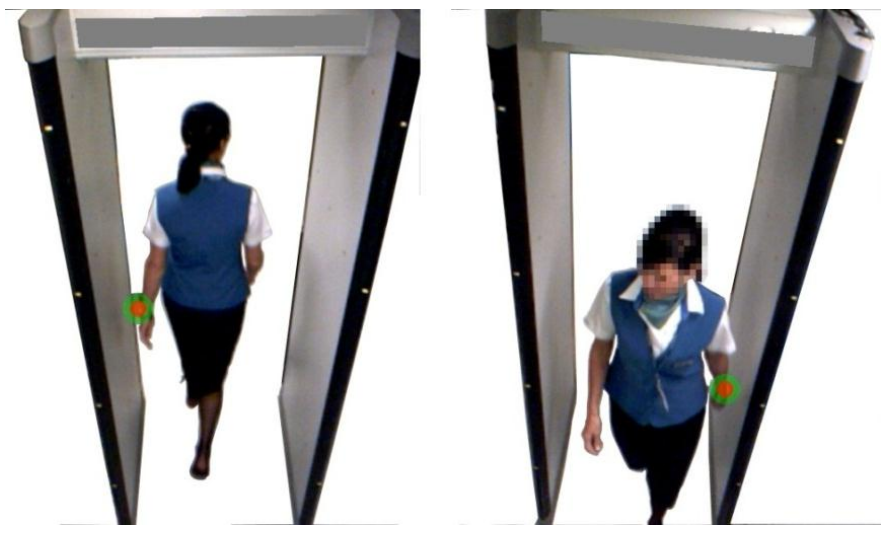

Figure 15. Example output from a system coupled with video technology

\section{Acknowledgements}

The authors would like to thank the UK Engineering and Physical Sciences Research Council (EPSRC) and the Technology Strategy Board (TSB) for financial support of this research under programmes DT/F002467 and TP M1649F respectively. The authors are also very grateful to Ari Visa and Juho Vihonen at Tampere University of Technology and Bill Lionheart at the University of Manchester for their encouragement and very useful suggestions during this research.

\section{References}

[1] Bell T H, Barrow B J and Miller J T 2001 Subsurface discrimination using electromagnetic induction sensors IEEE Trans. Geosci. Remote Sens.39 1286-1292

[2] Norton S J and Won I 2001 Identification of buried unexploded ordnance from broadband electromagnetic induction data IEEE Trans. Geosci. Remote Sens. 39 2253-2261

[3] Harrington R F 2001 Time Harmonic Magnetic Fields (New York : Wiley Interscience) pp 116-120

[4] Zhang Y, Collins M, Yu H, Baum C E and Carin L 2003 Sensing of unexploded ordinance with magnetometer and induction data: Theory and signal processing IEEE Trans. Geosci. Remote Sens.41 1005-1015

[5] Ulaby F T, Michielssen E and Umberto R 2010 Fundamentals of Applied Electrodynamics ed A Gilfillan (New Jersey : Prentice Hall) p 261

[6] Lorrain P, Corson D R and Lorrain F 1988 Electromagntic Fields and Waves (New York : W H Freeman and Co) p 424

[7] Kraus J D and Fleisch D A 1999 Electromagnetics with Applications (Singapore : McGraw-Hill) pp 96-98

[8] Lorrain P, Corson D R and Lorrain F 1988 Electromagnetic Fields and Waves (New York : W H Freeman and Co) pp 348-349

[9] Schwinger J, Deraad L L, Milton K A, Tsai W and Norton J 1998 Classical Electrodynamics (Boulder, CO : Westview Press) pp 325-326

[10] Tatum J B 2010 Electricity and Magnetism (Victoria, BC : University of Victoria) pp 1-2

[11] Dooley J W 2010 Macroscopic Physics and Thermodynamics (Millersville, PA : Millersville University)

[12] Lourakis M I A 2005 A brief description of the Levenberg-Marquardt algorithm implemented by levmar (Crete : Foundation forResearch and Technology)

[13] Gavin H 2011 The Levenberg-Marquadt method for nonlinear least suqares curve-fitting problems (Durham, NC : Duke University)

[14] Paulter N and Larson D 2009 NIJ Metal Detector Test Object Report (Gaithersburg, MD : National Institute for Justice)

[15] Tai C T 1997 Generalised Vector and Dyadic Analysis (Piscataway, NJ : IEEE Press) pp 8-10 